\title{
The Shape of Things to Come in Speech Production: A Functional Magnetic Resonance Imaging Study of Visual Form Interference during Lexical Access
}

\author{
Hanna S. Gauvin ${ }^{1}$, Katie L. McMahon ${ }^{1,2}$, Marcus Meinzer ${ }^{3}$, and Greig I. de Zubicaray ${ }^{1}$
}

\begin{abstract}
Studies of context effects in speech production have shown that semantic feature overlap produces interference in naming of categorically related objects. In neuroimaging studies, this semantic interference effect is consistently associated with involvement of left superior and middle temporal gyri. However, at least part of this effect has recently been shown to be attributable to visual form similarity, as categorically related objects typically share visual features. This fMRI study examined interference produced by visual form overlap in the absence of a category relation in a picture-word interference paradigm. Both visually similar and visually dissimilar distractors led to increased BOLD responses in the left inferior frontal gyrus compared with the congruent condition. Naming pictures in context with a dis-
\end{abstract}

\section{INTRODUCTION}

Speakers are influenced by the context in which their production occurs. For instance, in picture naming tasks, repeated access to a semantic category results in increased RTs (e.g., Kroll \& Stewart, 1994; Lupker, 1979; Rosinski, 1977). This "semantic interference effect" has been fundamental to the development of theories of lexical access during speech production.

Theoretical models of speech production differ with respect to how they explain the process of lexical selection and the architecture of the mental lexicon. The lexical-selection-by-competition model assumes that words are grouped into categories via connections to a higher order category node (e.g., "animal") and to nodes reflecting primitive features. According to this theory, the items attached to a common category node are represented as whole concepts, also referred to as "nondecomposed" semantic representations (e.g., Levelt, Roelofs, \& Meyer, 1999; Starreveld \& La Heij, 1995, 1996; Roelofs, 1992). During lexical selection, activation is assumed to increase for the target item and for the items that are connected to a common category node (e.g., other animals)

\footnotetext{
${ }^{1}$ Queensland University of Technology, ${ }^{2}$ Royal Brisbane \& Women's Hospital, ${ }^{3}$ The University of Queensland
}

tractor word denoting an object visually similar in form slowed RTs compared with unrelated words and was associated with reduced activity in the left posterior middle temporal gyrus. This area is reliably observed in lexical level processing during language production tasks. No significant differential activity was observed in areas typically engaged by early perceptual or conceptual feature level processing or in areas proposed to be engaged by postlexical language processes, suggesting that visual form interference does not arise from uncertainty or confusion during perceptual or conceptual identification or after lexical processing. We conclude that visual form interference has a lexical locus, consistent with the predictions of competitive lexical selection models. via coactivation. When multiple items from a semantic category have increased activation levels, either when repeatedly naming items from one category or by concurrent presentation of related targets and distractors, the resulting increase in activation of competing lexical candidates leads to a more effortful and time-consuming selection process (e.g., Belke, Meyer, \& Damian, 2005; Levelt et al., 1999; Starreveld \& La Heij, 1995, 1996; Roelofs, 1992; Glaser \& Glaser, 1989).

Decomposional models assume there is no representation of whole concepts at the semantic level; rather, there are multiple distributed feature nodes (e.g., "has a tail") directly connected to lexical representations. Semantic interference is proposed to arise as features are commonly shared between items within a semantic category, with activation spreading along the feature nodes' connections (e.g., Oppenheim, Dell, \& Schwartz, 2010; Howard, Nickels, Coltheart, \& Cole-Virtue, 2006; Goldrick \& Rapp, 2002; Caramazza, 1997; Dell \& O'Seaghdha, 1992). A third view proposes that lexical items are connected to both category nodes and feature nodes (Belke, 2013). Depending on the task, either shared class features or distinguishing features are activated. Some of the above models also view lexical selection as a noncompetitive process, in which the most highly activated target gets selected once activation exceeds a predetermined threshold or at a certain time 
point (e.g., Caramazza, 1997; Dell \& O'Seaghdha, 1992). As the latter accounts predict facilitation with increased activation because of semantic feature overlap, semantic interference is instead attributed to the operation of postlexical decision processes operating according to response-relevant criteria (e.g., category membership; Mahon, Costa, Peterson, Vargas, \& Caramazza, 2007). According to the response exclusion hypothesis, feature level information does not constitute a response-relevant criterion (see Mahon et al., 2007).

The role of visual form overlap between items during picture naming has until recently received little attention. In a visual world paradigm, Yee, Huffstetler, and Thompson-Schill (2011) demonstrated a fast coactivation of words sharing shape activation and somewhat slower coactivation of words sharing function. Such a fast and automatic coactivation has important implications for the interpretation of semantic interference effect; as there is more visual similarity among category coordinate items (e.g., cat and dog) than between categories (e.g., train and dog; e.g., Damian, Vigliocco, \& Levelt, 2001; La Heij, 1988), the observed semantic interference could thus at least partly reflect a confound because of visual similarity. Although many studies have attempted to address visual similarity by minimizing within-category visual similarities (e.g., Hocking, McMahon, \& de Zubicaray, 2009; Damian et al., 2001; La Heij, 1988), many categories unavoidably share some visual-structural features (buildings have windows and a roof, animals have faces, etc.), which are likely to be activated when presented in context.

Neumann and Kautz (1982; discussed in La Heij, 1988) were the first to report interference from form-related items that belonged to a different semantic category in picture naming. La Heij (1988) proposed that visual form interference might result from difficulties with object identification or from confusion arising from the printed distractor word denoting a visually similar concept. Recently, de Zubicaray et al. (2018) confirmed a visual form interference effect independent of categorical relations in the picture-word interference (PWI) task, in which participants name a target picture while ignoring a superimposed distractor word. Similar to semantic interference, form interference was observed reliably at short negative and zero SOAs. Results from a PWI psychological refractory period paradigm, in which participants need to respond sequentially to two presented stimuli associated with different tasks, yielded comparable results with the semantic psychological refractory period paradigm (Piai, Roelofs, Jensen, Schoffelen, \& Bonnefond, 2014). They concluded that the locus of visual form interference is likely to be central (i.e., lexical or later), rather than during early (prelexical) perceptual processes such as misidentification or identification difficulties.

To date, there is no clear answer to the question: What processes might produce visual form interference? Aside from misidentification or confusion at an early perceptual/conceptual level of processing, visual form interference might arise as activation spreads between shared visual features and their lexical representations. Another proposal is that interference arises at the lexical level by the creation of ad hoc (task-specific) categories (Abdel Rahman \& Melinger, 2009, 2011). Both possibilities are compatible with lexical-selection-by-competition accounts (e.g., Roelofs, 1992, 2001). Alternatively, interference might arise at the postlexical or self-monitoring level, if visual similarity is assumed to be a responserelevant exclusion criterion, which results in additional processing time for visually similar items (e.g., Mahon et al., 2007). However, this would require an ad hoc adaptation to the response exclusion account. A final possibility is that participants might be confused about whether the distractors refer to the target picture or visually similar distractor, but the visual confusion is expressed at the postlexical level.

The purpose of the current study was to clarify the locus of visual form interference effect in PWI with functional magnetic resonance imaging (fMRI). As each hypothesis makes different predictions about the recruitment of cortical areas, an fMRI study can provide pivotal information. If visual form interference arises at the conceptual-to-lexical level, we expect to observe reduced activation in middle and posterior portions of the left middle temporal gyrus (MTG) and superior temporal gyrus (STG), similar to activation patterns and areas reliably involved in lexical level processing (e.g., Acheson, Hamidi, Binder, \& Postle, 2011; Peeva et al., 2010; Indefrey \& Levelt, 2004). Signal reductions are reliably observed during fMRI studies of semantic interference in PWI when semantically related versus unrelated conditions are compared (Piai et al., 2014; de Zubicaray, Hansen, \& McMahon, 2013; Piai, Roelofs, Acheson, \& Takashima, 2013; de Zubicaray \& McMahon, 2009; see de Zubicaray \& Piai, 2019, for a review). A postlexical response exclusion account of visual form interference would predict recruitment of brain regions reliably correlated with postlexical stages of processing, such as phonetic encoding, syllabification, and monitoring, which have been ascribed to the (posterior) superior temporal cortex, premotor cortex, inferior frontal lobe, and ACC in various meta-analyses (Price, 2012; Indefrey, 2011; Indefrey \& Levelt, 2004). However, as none of the these regions is consistently observed across PWI studies, we cannot form any further hypotheses in terms of level of activation. In addition, Dhooge and Hartsuiker (2010) have proposed that a postlexical decision mechanism might reflect the operations of the verbal self-monitor, a role attributed to the STG by Indefrey and Levelt (2004; also Indefrey, 2011). If visual form interference arises because of visual confusion/misidentification (at either prelexical or postlexical levels), activation changes should be observed in areas implicated in visual processing or in processing feature overlap among objects such as the fusiform gyrus, lingual gyrus, and perirhinal cortex 
(e.g., Clarke, Taylor, Devereux, Randall, \& Tyler, 2013; Chan \& Downing, 2011; Hocking et al., 2009; Bussey, Saksida, \& Murray, 2005; Moss, Rodd, Stamatakis, Bright, \& Tyler, 2005; Cohen \& Dehaene, 2004; Tyler et al., 2004). Finally, we also compared incongruent (i.e., visually similar and dissimilar) versus congruent distractor conditions for which we expected to observe increased activation in ACC and inferior frontal cortex per previous studies of lexical interference (i.e., Stroop-like) effects (e.g., Piai et al., 2013; see de Zubicaray \& Piai, 2019, for a review).

\section{METHODS}

\section{Participants}

Twenty-one participants were recruited from the University of Queensland. Two participants had to be excluded from analysis because of head motion exceeding one voxel ( $3 \mathrm{~mm}$ in any direction) within an imaging run. The remaining 19 participants had a mean age of 24 (range $=18-37$ ) years and included nine women. All were right-handed and native English speakers, with no history of neurological or psychiatric disorder, substance dependence, or known hearing deficits. All had normal or corrected-to-normal vision and gave informed consent. The study was approved by the ethical review committee of the University of Queensland. Participants were reimbursed AUD 30 for participating.

\section{Stimulus Material and Task Design}

Participants performed a PWI task in which they named a picture while ignoring a distractor word that was congruent with the picture name (i.e., identical) or denoted a visually similar or visually dissimilar object. An illustration of the three conditions is provided in Figure 1. Stimulus material and task design were identical to the visual form PWI Experiment 1 of de Zubicaray et al. (2018) but used a single SOA of 0 as visual interference was reliably observed at this SOA and an intertrial interval of $15 \mathrm{sec}$ to accommodate the fMRI acquisition protocol. Participants were familiarized with the target stimuli in the scanner bore, after positioning and before data acquisition. Stimuli material and scripts are publicly available via Open Science Framework (osf.io/dc2v8/).

\section{Apparatus}

Stimuli presentation, response recording, and latency measurement (i.e., voice key) were accomplished via the Cogent 2000 toolbox extension (www.vislab.ucl.ac. uk/cogent_2000.php) for MATLAB (2010a; MathWorks, Inc.) via a personal computer, a rear-positioned projector screen, and an MR-compatible, fiber-optic dual-channel noise-canceling microphone attached to the head coil (FOMRI-III, Optoacoustics Ltd.; www.optoacoustics. com). Participants viewed back-projected stimuli via a mirror positioned on the head coil. Pictures subtended approximately $10^{\circ}$ of visual angle when each participant was positioned for scanning. A 30-dB attenuating headset was used to reduce gradient noise. Response latencies were determined online by Cogent 2000, and the digital audio files were inspected offline (i.e., postexperiment) using Audacity software (audacity.sourceforge.net) to verify whether latencies reflected naming responses or nonvocal noise.

\section{Image Acquisition}

Images were acquired using a 3-T Siemens MAGNETOM Trio TIM (Siemens Medical Systems) with a 12-channel head coil. Nine BOLD gradient-echo EPI volumes were acquired across two consecutive sessions (36 slices, acquisition time $=3 \mathrm{sec}$, repetition time $=15,000 \mathrm{msec}$, echo time $=36 \mathrm{msec}, 64 \times 64$ matrix, $3.3 \mathrm{~mm} \times 3.3 \mathrm{~mm}$ in-plane resolution, $3-\mathrm{mm}$ slice with $0.3-\mathrm{mm}$ gap, and a flip angle of $80^{\circ}$ ). The first two volumes were discarded. Behavioral trials were interleaved with image acquisition using a sparse temporal sampling design (Gracco, Tremblay, \& Pike, 2005). This allows overt verbal responses to be made during a scanner off-phase to avoid articulation-related artifacts. For each trial, no field gradients were applied for a 4-sec period of relative silence allowing for stimuli to be presented and recording of the participant's overt verbal response and then immediately applied for image acquisition. A single-image volume was then acquired within $3 \mathrm{sec}$, approximately coincident with the picture naming trial's estimated peak BOLD response. No field gradients were applied for an additional 8-sec period to allow the BOLD response to the gradient noise to return to baseline (for a diagram of the imaging protocol, see Figure 2). A 3-D T1-weighted structural image was acquired with an magnetization
Figure 1. Examples of the target pictures and distractor words (in red) in the three presentation conditions (printed below in black) of the PWI task

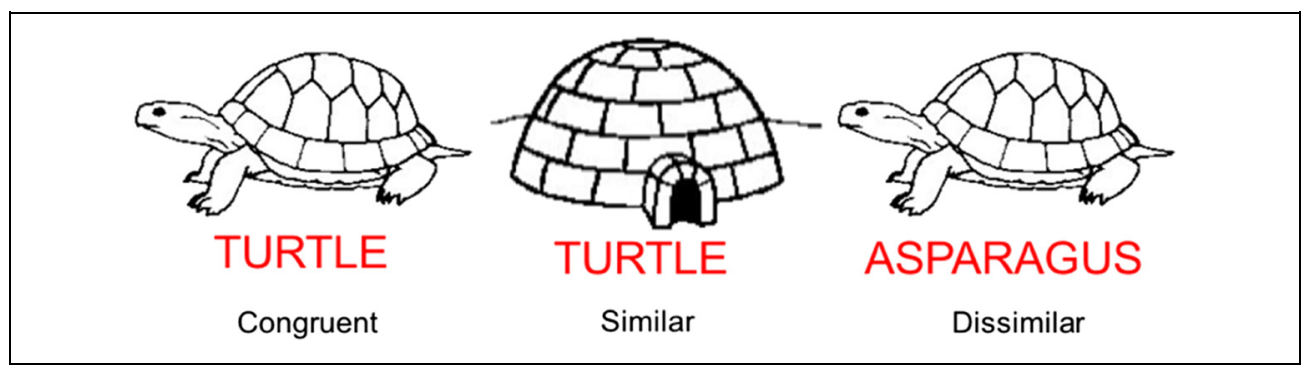


Figure 2. Example of a single trial from the sparse imaging acquisition protocol (adapted from de Zubicaray, Wilson, McMahon, \& Muthiah, 2001).

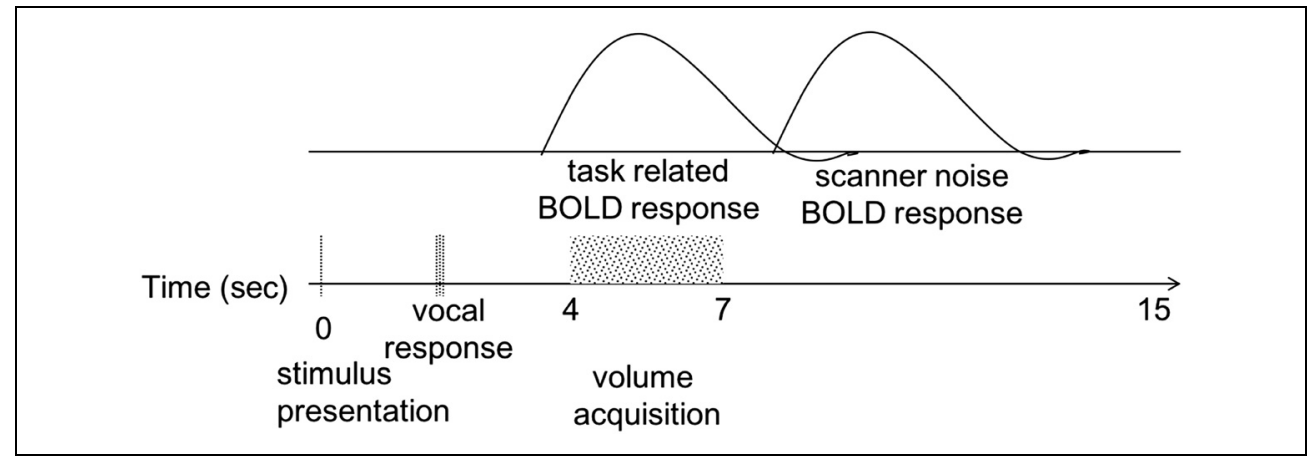

prepared rapid gradient echo sequence (1-mm isotropic voxel).

\section{Data Analysis}

\section{fMRI Data Preprocessing}

The fMRI data were preprocessed and analyzed using SPM software (SPM12; Wellcome Department of Imaging Neuroscience). Images were realigned to the first image of the first block using the INRIAlign toolbox (Freire, Roche, \& Mangin, 2002). A mean image was generated from the realigned series and coregistered to the T1weighted image. Using the Segment procedure, the T1weighted image was segmented. The DARTEL toolbox (Ashburner, 2007) was used to create a custom group template from the segmented gray and white matter images. Individual flow fields were then used to normalize the realigned fMRI volumes to the Montreal Neurological Institute atlas $\mathrm{T} 1$ template. The images were resampled to $3 \times 3 \times 3 \mathrm{~mm}$ isotropic voxels and smoothed with a 9-mm FWHM isotropic Gaussian kernel to accommodate the lower signal-to-noise ratio associated with sparse acquisitions (Nebel et al., 2005). Global signal effects were estimated and removed using a voxel level linear model (Macey, Macey, Kumar, \& Harper, 2004).

A two-stage, mixed effects statistical analysis was conducted. Event types corresponding to the three distractor types and an error condition were modeled as effects of interest, with delta functions representing each picture onset, and convolved with a basis function consisting of a single finite impulse response with a window length corresponding to the acquisition time. As the sparse image sequence does not acquire BOLD time course information, trials were not convolved with a conventional hemodynamic response function (see Gracco et al., 2005; Eden, Joseph, Brown, Brown, \& Zeffiro, 1999; Elliott, Bowtell, \& Morris, 1999). Linear contrasts were applied to each participant's parameter estimates at the fixed effects level and then entered in a group-level, randomeffects, repeated-measures ANOVA in which covariance components were estimated using a restricted maximum likelihood procedure to correct for nonsphericity (Friston et al., 2002). Planned $t$ contrasts were employed within the SPM12 random effects design matrix to identify regions showing significant differences in activity between the three experimental conditions.

As we had a priori hypotheses implicating specific brain regions/mechanisms in visual interference, we predefined ROIs for analysis via small volume corrections based largely on the updated Indefrey (2011) metaanalysis of brain regions involved in spoken word production. A priori ROIs were defined using labeled maximum likelihood gray matter maps from 3-D probabilistic atlases to test prelexical/perceptual, lexical, and postlexical mechanisms (Holdstock, Hocking, Notley, Devlin, \& Price, 2009; Hammers et al., 2003). Selected ROIs were the following left hemisphere regions: midMTG (lexical-semantic processing; e.g., Acheson et al., 2011; Indefrey, 2011), posterior MTG (pMTG)/STG (word form processing; e.g., Indefrey \& Levelt, 2004), STG (verbal self-monitoring; e.g., Indefrey, 2011), inferior frontal gyrus (IFG) and premotor cortex (postlexical syllabification and phonetic encoding; e.g., Price, 2012; Indefrey, 2011; in addition, the IFG is proposed to be involved in lexical selection; e.g., Belke \& Stielow, 2013; Schnur et al., 2009), ACC (conflict monitoring; e.g., Gauvin, De Baene, Brass, \& Hartsuiker, 2016; Piai et al., 2013; Indefrey, 2011; de Zubicaray et al., 2001), perirhinal cortex (shared semantic feature activation and priming; e.g., Hocking et al., 2009; Tyler et al., 2004), and fusiform and lingual gyri (visual processing; e.g., Hocking, McMahon, \& de Zubicaray, 2010; de Zubicaray, McMahon, Eastburn, \& Pringle, 2008; Martin \& Chao, 2001). In addition to the ROI analyses with small volume correction, we conducted an exploratory whole-brain analysis. A height threshold of $p<.001$ was adopted in conjunction with a cluster FWE-corrected threshold of $p<.05$ estimated for the whole brain and for each ROI.

\section{RESULTS}

\section{Behavioral Data}

Because of excessive head movement during image acquisition, defined as exceeding one voxel $(3 \mathrm{~mm})$ within an imaging run, data from two participants were excluded from both behavioral and fMRI analyses. Of the 
Figure 3. Mean picture naming latencies per distractor condition.

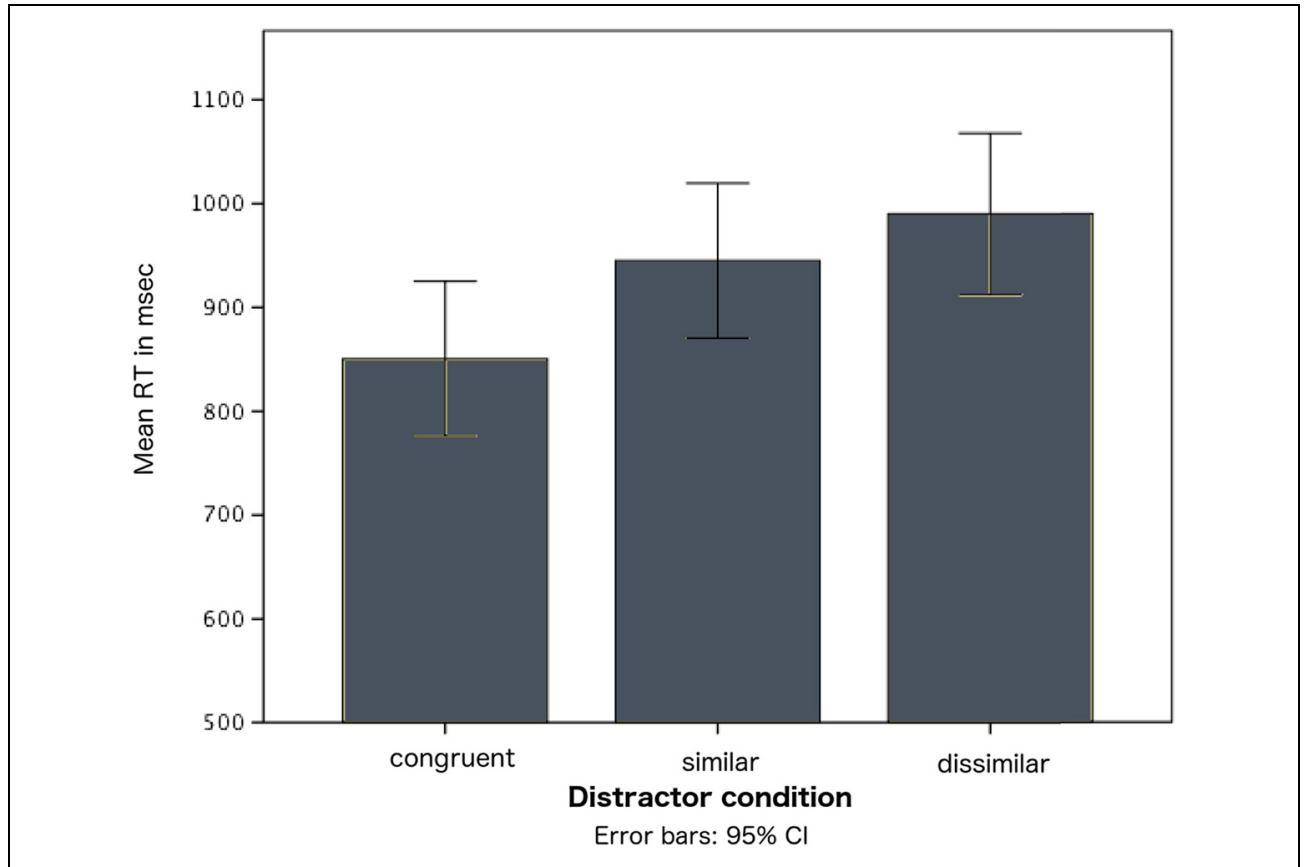

remaining data, the following trials were removed before data analysis: trials on which the voice key failed to detect a response $(n=67,3.9 \%)$, trials with an incorrect naming response $(n=42,2.5 \%)$, and trials with an RT below 350 msec or above 2000 msec ( $n=7,0.4 \%$ ). In addition, trials were removed in which the RT deviated more than 2.5 SDs from each participant's mean within condition $(n=36,2.1 \%)$. In total, 1,558 trials were taken into analysis (8.9\% data loss).

To test whether visual form distractor relations affected picture naming RTs, repeated-measures ANOVAs were performed in SPSS 24. A significant main effect of Distractor was observed for both the by-participants $\left(F_{1}\right)$ and by-items $\left(F_{2}\right)$ analyses, $F_{1}(2,36)=34.55, p<$
$.001, \eta_{\mathrm{p}}^{2}=.65$, and $F_{2}(2,58)=77.58, p<.001, \eta_{\mathrm{p}}^{2}=$ .73). Paired $t$ tests (by-participants $t_{1}$ and by-items $t_{2}$ ) showed that RTs were significantly faster for items paired with congruent distractors (mean RT $=851 \mathrm{msec}$, 95\% CI $[777,925])$ compared with dissimilar distractors (mean RT $=945 \mathrm{msec}$, 95\% CI [871, 1020]; 95-msec difference: $t_{1}(18)=5.30, p<.001, d=0.61$, and $t_{2}(29)=$ $8.51, p<.001, d=1.50$ ) and similar distractors (mean $\mathrm{RT}=990 \mathrm{msec}, 95 \% \mathrm{CI}[912,1068] ; 139$-msec difference: $t_{1}(18)=8.21, p<.001, d=0.88$, and $t_{2}(29)=$ $14.78, p<.001, d=2.02$ ). Crucially, a significant visual interference effect of $45 \mathrm{msec}$ was observed; responses to items paired with visually similar distractors were named slower compared with items paired with visually
Figure 4. Visual form interference (dissimilar > similar), left-hemisphere surface rendering of the significant activation cluster in the pMTG (FWE, $p<.05$ ).

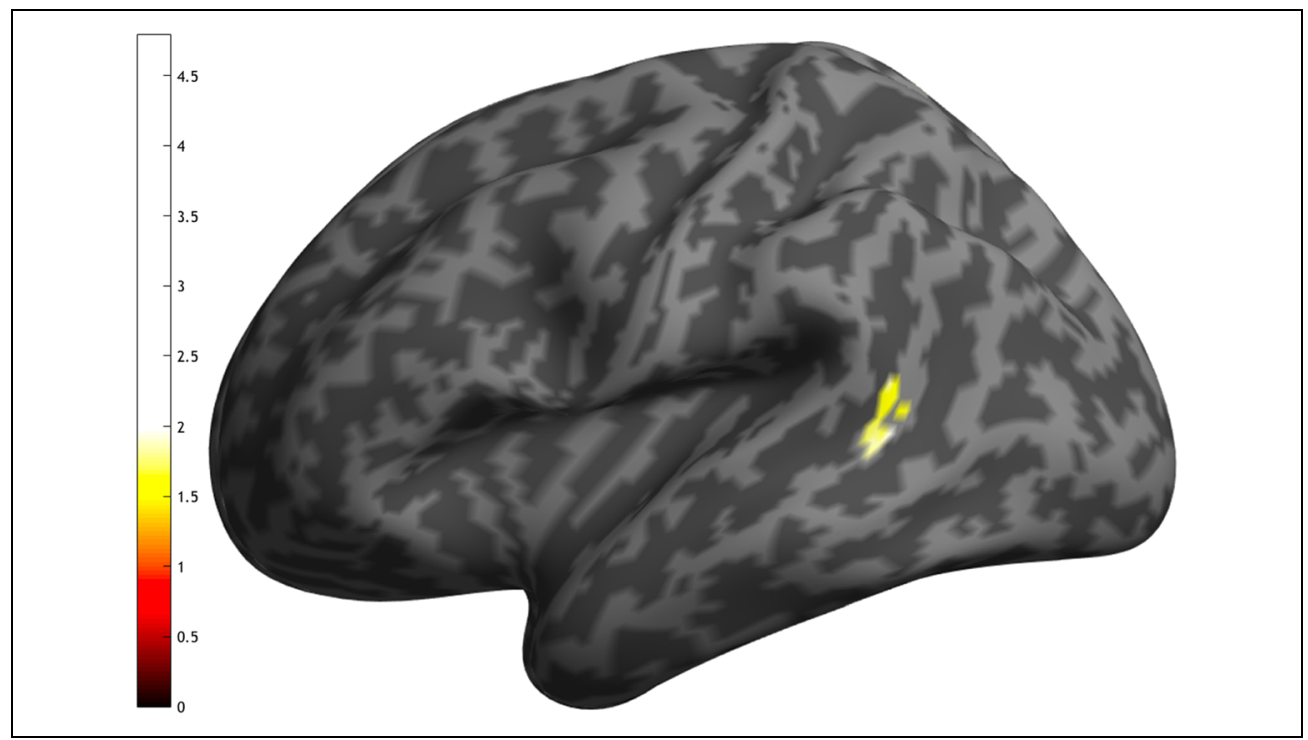


Figure 5. Lexical interference (dissimilar > congruent), left-hemisphere surface rendering of the significant activation cluster in the IFG and middle occipital gyrus (FWE, $p<.05)$

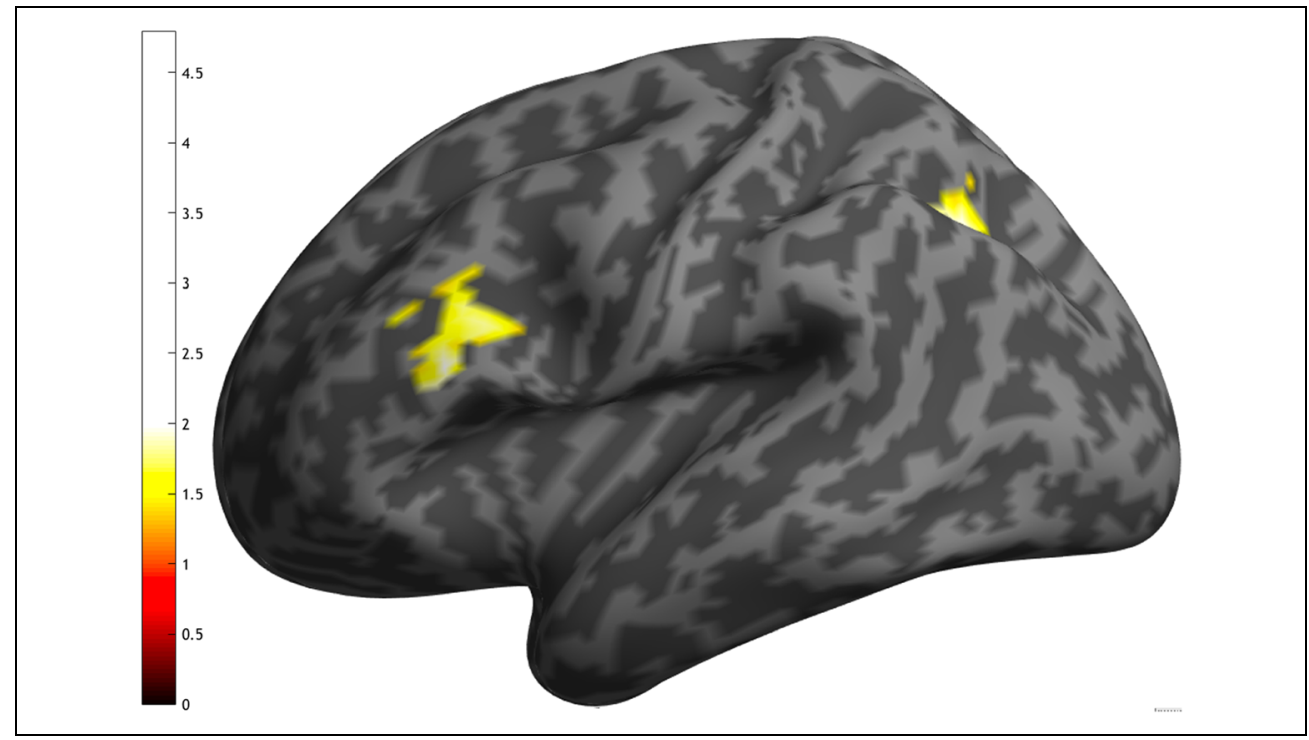

dissimilar distractors, $t_{1}(18)=2.72, p=.014, d=0.28$, and $t_{2}(29)=3.51, p=.001, d=0.066$. Mean RTs per distractor condition are presented in Figure 3.

\section{fMRI Data}

Group-averaged motion and rotation parameters from the remaining 19 participants were less than $1 \mathrm{~mm}$ and $1^{\circ}$, respectively, consistent with data reported for sparse fMRI acquisitions in the literature (e.g., Gracco et al., 2005).

\section{A priori Defined ROI Analyses}

Contrasts of the visually similar and dissimilar conditions revealed only a significantly decreased BOLD signal response in the posterior middle temporal lobe ROI (dissimilar > similar; see Figure 4). Compared with the congruent condition, both the dissimilar (dissimilar $>$ congruent) and similar (similar $>$ congruent) conditions revealed significantly increased responses in the middle occipital gyrus and in the IFG, with a peak in the pars triangularis (see Figure 5). None of the other ROIs or contrasts showed significant activation clusters. An overview of the significant activity is represented in Table 1.

\section{Exploratory Whole-Brain Analyses}

A comparison of the three distractor conditions across the whole brain revealed a significant increased response for the similar > congruent contrast in the IFG, with a peak in the pars triangularis (see Table 1). No other

Table 1. Cerebral Regions Showing Significant Activation as a Function of the Visual Similarity of the Distractor Condition

\begin{tabular}{|c|c|c|c|}
\hline Contrasts & Peak MNI $(x, y, z)$ & Z Score & Cluster Size (Voxels) \\
\hline \multicolumn{4}{|l|}{ Dissimilar $>$ Similar } \\
\hline Left $\mathrm{pMTG}^{\mathrm{a}}$ & $-60,-50,2$ & 3.94 & 91 \\
\hline \multicolumn{4}{|l|}{ Dissimilar $>$ Congruent } \\
\hline Left IFG (pars triangularis) ${ }^{\mathrm{a}}$ & $-48,24,22$ & 4.85 & 454 \\
\hline Left middle occipital gyrus ${ }^{\mathrm{a}}$ & $-30,-64,36$ & 4.09 & 174 \\
\hline \multicolumn{4}{|l|}{ Similar $>$ Congruent } \\
\hline Left IFG (pars triangularis) ${ }^{\mathrm{a}, \mathrm{b}}$ & $-46,24,24$ & 5.42 & 791 \\
\hline Left middle occipital gyrus ${ }^{\mathrm{a}}$ & $-26,-64,34$ & 4.92 & 502 \\
\hline
\end{tabular}

MNI $=$ Montreal Neurological Institute.

${ }^{\mathrm{a}} \mathrm{ROI}$ corrected.

${ }^{\mathrm{b}}$ Whole-brain corrected. 
significant BOLD responses were observed for any of the other contrasts.

\section{DISCUSSION}

This is the first study to report fMRI findings for visual form interference during spoken word production in the absence of a categorical relation. The form interference effect in naming latencies reported by de Zubicaray et al. (2018) was replicated with identical stimuli, revealing a reduced BOLD signal response in the left pMTG for visually similar items compared with visually dissimilar items. However, we failed to observe significant differential activity in brain areas typically associated with visual processing/confusion or in areas associated with postlexical processes in speech production.

\section{Visual Form Interference}

The visual form interference effect was associated with a reduced BOLD signal response for visually similar items compared with visually dissimilar items in the left pMTG. The pMTG is reliably observed in functional neuroimaging studies of lexical-semantic processing (Binder, Desai, Graves, \& Conant, 2009; Vigneau et al., 2006; Indefrey \& Levelt, 2004; Cabeza \& Nyberg, 2000). Importantly, the pMTG is also reliably observed in neuroimaging studies of semantic interference, where the activation reductions have been attributed to lateral inhibition mechanisms and reduced effort involved in lexical retrieval in the related and congruent conditions, respectively, compared with the unrelated condition (Piai et al., 2013, 2014; de Zubicaray et al., 2013; de Zubicaray \& McMahon, 2009). Thus, it is likely that visual form interference also arises at the conceptual-to-lexical level, reflecting the spread of activation from an ad hoc, task-specific category created among response set members (Abdel Rahman \& Melinger, 2009, 2011). Given the similarities between visual form interference and semantic interference, both in terms of production latencies and in neural response, it seems highly plausible that the semantic interference effect reported in previous fMRI studies to some extent reflects visual interference because of visual form similarity of items within the same semantic category.

We reasoned that, if visual form interference results from prelexical or postlexical visual confusion/misidentification, differential activation would be observed in areas related to visual processing, such as the fusiform gyrus, lingual gyrus, and perirhinal cortex (e.g., Clarke et al., 2013; Chan \& Downing, 2011; Hocking et al., 2009; Bussey et al., 2005; Moss et al., 2005; Cohen \& Dehaene, 2004; Tyler et al., 2004). We also reasoned that a postlexical locus for visual form interference attributable to a decision mechanism acting on well-formed responses in an articulatory buffer (e.g., Dhooge \& Hartsuiker, 2010; Mahon et al., 2007) would have resulted in differential activation in areas related to syllabification and phonetic encoding (e.g., Price, 2012; Indefrey, 2011) and/or selfmonitoring, such as ACC or STG (Indefrey, 2011; Dhooge \& Hartsuiker, 2010; Indefrey \& Levelt, 2004). As none of these regions showed significant differential activity, the data from the current study do not support a prelexical locus of visual form interference or a postlexical one.

\section{Lexical Interference Effect}

Compared with the congruent condition, both similar and dissimilar distractors lead to an increased response in the left IFG, specifically in the pars triangularis. The activation corresponds well with the suggested role for the left IFG, and pars triangularis specifically, in resolving competition between competing lexical items (Badre \& Wagner, 2007; Moss et al., 2005; Kan \& ThompsonSchill, 2004; but see Piai \& Knight, 2018, and Piai, Riès, \& Swick, 2016). Alternatively, the pars triangularis is consistently observed in word retrieval neuroimaging studies, so within that framework, the activation could also be interpreted as reflecting more effortful word retrieval during picture naming in the presence of a distractor word (see Price, 2010, 2012). In addition, increased activity in the middle occipital gyrus was observed for both similar and dissimilar distractors when compared with the congruent condition. This might indicate that visual object recognition is less effortful when the picture is accompanied by its name, that is, identity priming. The observed activations complement the behavioral data, which show longer RTs for related and unrelated distractors compared with the congruent condition.

\section{Conclusions}

We observed a significant visual interference effect in naming when pictures were paired with visually similar compared with visually dissimilar distractor words, independent of a category coordinate relation. The fMRI data support the notion that visual form interference reflects conceptual-to-lexical processing, a role ascribed to pMTG. Moreover, the observed pMTG activity bears a striking resemblance to that observed during semantic interference in PWI. Thus, behavioral and fMRI reports of semantic interference effects in production are likely to at least reflect a component of visual interference. Importantly, the findings demonstrate that visual form interference is unlikely to arise from confusion at the visual processing level, at either prelexical or postlexical levels, or arise during postlexical scrutiny of responses in an articulatory buffer. Finally, the current study demonstrates that lexical selection can be influenced by a single feature in the absence of a category relation.

\section{Acknowledgments}

This research was supported by Australian Research Council Discovery Project grant DP150103997. 
Reprint requests should be sent to Hanna S. Gauvin, Faculty of Health, Department of Psychology \& Counselling, Queensland University of Technology, O Block B Wing level 5, Ring Road, Brisbane, QLD 4059, Australia, or via e-mail: hanna.gauvin@ qut.edu.au.

\section{REFERENCES}

Abdel Rahman, R., \& Melinger, A. (2009). Semantic context effects in language production: A swinging lexical network proposal and a review. Language and Cognitive Processes, 24, 713-734.

Abdel Rahman, R., \& Melinger, A. (2011). The dynamic microstructure of speech production: Semantic interference built on the fly. Journal of Experimental Psychology: Learning, Memory, and Cognition, 37, 149-161.

Acheson, D. J., Hamidi, M., Binder, J. R., \& Postle, B. R. (2011). A common neural substrate for language production and verbal working memory. Journal of Cognitive Neuroscience, 23, 1358-1367.

Ashburner, J. (2007). A fast diffeomorphic image registration algorithm. Neuroimage, 38, 95-113.

Badre, D., \& Wagner, A. D. (2007). Left ventrolateral prefrontal cortex and the cognitive control of memory.

Neuropsychologia , 45, 2883-2901.

Belke, E. (2013). Long-lasting inhibitory semantic context effects on object naming are necessarily conceptually mediated: Implications for models of lexical-semantic encoding. Journal of Memory and Language, 69, 228-256.

Belke, E., Meyer, A. S., \& Damian, M. F. (2005). Refractory effects in picture naming as assessed in a semantic blocking paradigm. Quarterly Journal of Experimental Psychology, 58A, 667-692.

Belke, E., \& Stielow, A. (2013). Cumulative and non-cumulative semantic interference in object naming: Evidence from blocked and continuous manipulations of semantic context. Quarterly Journal of Experimental Psychology, 66, 2135-2160.

Binder, J. R., Desai, R. H., Graves, W. W., \& Conant, L. L. (2009). Where is the semantic system? A critical review and metaanalysis of 120 functional neuroimaging studies. Cerebral Cortex, 19, 2767-2796.

Bussey, T. J., Saksida, L. M., \& Murray, E. A. (2005). The perceptualmnemonic/feature conjunction model of perirhinal cortex function. Quarterly Journal of Experimental Psychology, 58B, 269-282.

Cabeza, R., \& Nyberg, L. (2000). Imaging cognition II: An empirical review of 275 PET and fMRI studies. Journal of Cognitive Neuroscience, 12, 1-47.

Caramazza, A. (1997). How many levels of processing are there in lexical access? Cognitive Neuropsychology, 14, 177-208.

Chan, A. W.-Y., \& Downing, P. E. (2011). Faces and eyes in human lateral prefrontal cortex. Frontiers in Human Neuroscience, $5,51$.

Clarke, A., Taylor, K. I., Devereux, B., Randall, B., \& Tyler, L. K. (2013). From perception to conception: How meaningful objects are processed over time. Cerebral Cortex, 23, 187-197.

Cohen, L., \& Dehaene, S. (2004). Specialization within the ventral stream: The case for the visual word form area. Neuroimage, 22, 466-476.

Damian, M. F., Vigliocco, V., \& Levelt, W. J. M. (2001). Effects of semantic context in the naming of pictures and words. Cognition, 81, B77-B86.

de Zubicaray, G. I., Hansen, S., \& McMahon, K. L. (2013). Differential processing of thematic and categorical conceptual relations in spoken word production. Journal of Experimental Psychology: General, 142, 131-142.

de Zubicaray, G. I., McLean, M., Oppermann, F., Hegarty, A., McMahon, K. L., \& Jescheniak, J. D. (2018). The shape of things to come in speech production: Visual form interference during lexical access. Quarterly Journal of Experimental Psychology, 71, 1921-1938.

de Zubicaray, G. I., \& McMahon, K. L. (2009). Auditory context effects in picture naming investigated with event-related fMRI. Cognitive, Affective \& Behavioral Neuroscience, 9, 260-269.

de Zubicaray, G. I., McMahon, K. L., Eastburn, M. M., \& Pringle, A. J. (2008). Negative priming in naming of categorically related objects: An fMRI study. Cortex, 44, 881-889.

de Zubicaray, G. I., \& Piai, V. (2019). Investigating the spatial and temporal components of speech production. In G. I. de Zubicaray \& N. O. Schiller (Eds.), The Oxford handbook of neurolinguistics. Oxford, UK: Oxford University Press.

de Zubicaray, G. I., Wilson, S. J., McMahon, K. L., \& Muthiah, S. B. (2001). The semantic interference effect in the pictureword paradigm: An event-related fMRI study employing overt responses. Human Brain Mapping, 14, 218-227.

Dell, G. S., \& O'Seaghdha, P. G. (1992). Stages of lexical access in language production. Cognition, 42, 287-314.

Eden, G. F., Joseph, J. E., Brown, H. E., Brown, C. P., \& Zeffiro, T. A. (1999). Utilizing hemodynamic delay and dispersion to detect fMRI signal change without auditory interference: The behavioral interleaved gradients technique. Magnetic Resonance in Medicine, 41, 13-20.

Elliott, M. R., Bowtell, R. W., \& Morris, P. G. (1999). The effect of scanner sound in visual, motor, and auditory functional MRI. Magnetic Resonance in Medicine, 41, 1230-1235.

Freire, L., Roche, A., \& Mangin, J.-F. (2002). What is the best similarity measure for motion correction in fMRI time series? IEEE Transactions on Medical Imaging, 21, 470-484.

Friston, K. J., Glaser, D. E., Henson, R. N. A., Kiebel, S., Phillips, C., \& Ashburner, J. (2002). Classical and Bayesian inference in neuroimaging: Applications. Neuroimage, 16, 484-512.

Gauvin, H. S., De Baene, W., Brass, M., \& Hartsuiker, R. J. (2016). Conflict monitoring in speech processing: An fMRI study of error detection in speech production and perception. Neuroimage, 126, 96-105.

Glaser, W. R., \& Glaser, M. O. (1989). Context effects in Strooplike word and picture processing. Journal of Experimental Psychology: General, 118, 13-42

Goldrick, M., \& Rapp, B. (2002). A restricted interaction account (RIA) of spoken word production: The best of both worlds. Aphasiology, 16, 20-55.

Gracco, V. L., Tremblay, P., \& Pike, B. (2005). Imaging speech production using fMRI. Neuroimage, 26, 294-301.

Hammers, A., Allom, R., Koepp, M. J., Free, S. L., Myers, R., Lemieux, L., et al. (2003). Three-dimensional maximum probability atlas of the human brain, with particular reference to the temporal lobe. Human Brain Mapping, 19, 224-247.

Hocking, J., McMahon, K. L., \& de Zubicaray, G. I. (2009). Semantic context and visual feature effects in object naming: An fMRI study using arterial spin labeling. Journal of Cognitive Neuroscience, 21, 1571-1583.

Hocking, J., McMahon, K. L., \& de Zubicaray, G. I. (2010). Semantic interference in object naming: An fMRI study of the postcue naming paradigm. Neuroimage, 50, 796-801.

Holdstock, J. S., Hocking, J., Notley, P., Devlin, J. T., \& Price, C. J. (2009). Integrating visual and tactile information in the perirhinal cortex. Cerebral Cortex, 19, 2993-3000.

Howard, D., Nickels, L., Coltheart, M., \& Cole-Virtue, J. (2006). Cumulative semantic inhibition in picture naming: Experimental and computational studies. Cognition, 100, 464-482.

Indefrey, P. (2011). The spatial and temporal signatures of word production components: A critical update. Frontiers in Psychology, 2, 255. 
Indefrey, P., \& Levelt, W. J. M. (2004). The spatial and temporal signatures of word production components. Cognition, 92, 101-144.

Kan, I. P., \& Thompson-Schill, S. L. (2004). Selection from perceptual and conceptual representations. Cognitive, Affective \& Behavioral Neuroscience, 4, 466-482.

Kroll, J. F., \& Stewart, E. (1994). Category interference in translation and picture naming: Evidence for asymmetric connections between bilingual memory representations. Journal of Memory and Language, 33, 149-174.

La Heij, W. (1988). Components of Stroop-like interference in picture naming. Memory \& Cognition, 16, 400-410.

Levelt, W. J. M., Roelofs, A., \& Meyer, A. S. (1999). A theory of lexical access in speech production. Behavioral and Brain Sciences, 22, 1-75.

Lupker, S. J. (1979). The semantic nature of response competition in the picture-word interference task. Memory \& Cognition, 7, 485-495.

Macey, P. M., Macey, K. E., Kumar, R., \& Harper, R. M. (2004). A method for removal of global effects from fMRI time series. Neuroimage, 22, 360-366.

Mahon, B. Z., Costa, A., Peterson, R., Vargas, K. A., \& Caramazza, A. (2007). Lexical selection is not by competition: A reinterpretation of semantic interference and facilitation effects in the picture-word interference paradigm. Journal of Experimental Psychology: Learning, Memory, and Cognition, 33, 503-535.

Martin, A., \& Chao, L. L. (2001). Semantic memory and the brain: Structure and processes. Current Opinion in Neurobiology, 11, 194-201.

Moss, H. E., Rodd, J. M., Stamatakis, E. A., Bright, P., \& Tyler, L. K. (2005). Anteromedial temporal cortex supports fine-grained differentiation among objects. Cerebral Cortex, 15, 616-627.

Nebel, K., Stude, P., Wiese, H., Müller, B., de Greiff, A., Forsting, M., et al. (2005). Sparse imaging and continuous eventrelated fMRI in the visual domain: A systematic comparison. Human Brain Mapping, 24, 130-143.

Neumann, O., \& Kautz, L. (1982). Semantische förderung und semantische interferenz im benennungsexperiment

[Semantic facilitation and semantic interference in a naming experiment] (Bericht Nr. 23/1982) [Report No. 23/1982]. Bochum, Germany: University of Bochum.

Oppenheim, G. M., Dell, G. S., \& Schwartz, M. F. (2010). The dark side of incremental learning: A model of cumulative semantic interference during lexical access in speech production. Cognition, 114, 227-252.

Peeva, M. G., Guenther, F. H., Tourville, J. A., Nieto-Castanon, A., Anton, J.-L., Nazarian, B., et al. (2010). Distinct representations of phonemes, syllables, and supra-syllabic sequences in the speech production network. Neuroimage, 50, 626-638.
Piai, V., \& Knight, R. T. (2018). Lexical selection with competing distractors: Evidence from left temporal lobe lesions. Psychonomic Bulletin \& Review, 25, 710-717.

Piai, V., Riès, S. K., \& Swick, D. (2016). Lesions to lateral prefrontal cortex impair lexical interference control in word production. Frontiers in Human Neuroscience, 9, 721.

Piai, V., Roelofs, A., Acheson, D. J., \& Takashima, A. (2013). Attention for speaking: Domain-general control from the anterior cingulate cortex in spoken word production. Frontiers in Human Neuroscience, 7, 832.

Piai, V., Roelofs, A., Jensen, O., Schoffelen, J.-M., \& Bonnefond, M. (2014). Distinct patterns of brain activity characterise lexical activation and competition in spoken word production. PLoS One, 9, e88674.

Price, C. J. (2010). The anatomy of language: A review of 100 fMRI studies published in 2009. Annals of the New York Academy of Sciences, 1191, 62-88.

Price, C. J. (2012). A review and synthesis of the first 20 years of PET and fMRI studies of heard speech, spoken language and reading. Neuroimage, 62, 816-847.

Roelofs, A. (1992). A spreading-activation theory of lemma retrieval in speaking. Cognition, 42, 107-142.

Roelofs, A. (2001). Set size and repetition matter: Comment on Caramazza and Costa (2000). Cognition, 80, 283-290.

Rosinski, R. R. (1977). Picture-word interference is semantically based. Child Development, 48, 643-647.

Schnur, T. T., Schwartz, M. F., Kimberg, D. Y., Hirshorn, E., Coslett, H. B., \& Thompson-Schill, S. L. (2009). Localizing interference during naming: Convergent neuroimaging and neuropsychological evidence for the function of Broca's area. Proceedings of the National Academy of Sciences, U.S.A., 106, 322-327.

Starreveld, P. A., \& La Heij, W. (1995). Semantic interference, orthographic facilitation, and their interaction in naming tasks. Journal of Experimental Psychology: Learning, Memory, and Cognition, 21, 686-698.

Starreveld, P. A., \& La Heij, W. (1996). Time-course analysis of semantic and orthographic context effects in picture naming. Journal of Experimental Psychology: Learning, Memory, and Cognition, 22, 896-918.

Tyler, L. K., Stamatakis, E. A., Bright, P., Acres, K., Abdallah, S., Rodd, J. M., et al. (2004). Processing objects at different levels of specificity. Journal of Cognitive Neuroscience, 16, 351-362.

Vigneau, M., Beaucousin, V., Hervé, P. Y., Duffau, H., Crivello, F., Houdé, O., et al. (2006). Meta-analyzing left hemisphere language areas: Phonology, semantics, and sentence processing. Neuroimage, 30, 1414-1432.

Yee, E., Huffstetler, S., \& Thompson-Schill, S. L. (2011). Function follows form: Activation of shape and function features during object identification. Journal of Experimental Psychology: General, 140, 348-363. 